\title{
Aa. Vv., Esculape et Dionysos. Mélanges en l'honneur de Jean Céard
}

\section{Michele Mastroianni}

\section{Q OpenEdition \\ 1 Journals}

\section{Edizione digitale}

URL: http://journals.openedition.org/studifrancesi/8200

DOI: $10.4000 /$ studifrancesi.8200

ISSN: 2421-5856

\section{Editore}

Rosenberg \& Sellier

\section{Edizione cartacea}

Data di pubblicazione: 1 mai 2009

Paginazione: 154-155

ISSN: 0039-2944

\section{Notizia bibliografica digitale}

Michele Mastroianni, «Aa. Vv., Esculape et Dionysos. Mélanges en l'honneur de Jean Céard», Studi

Francesi [Online], 157 (LIII | I) | 2009, online dal 30 novembre 2015, consultato il 08 janvier 2021. URL: http://journals.openedition.org/studifrancesi/8200 ; DOI: https://doi.org/10.4000/studifrancesi.8200

Questo documento è stato generato automaticamente il 8 janvier 2021.

\section{(c) (i) $\odot$}

Studi Francesi è distribuita con Licenza Creative Commons Attribuzione - Non commerciale - Non opere derivate 4.0 Internazionale. 


\title{
Aa. Vv., Esculape et Dionysos. Mélanges en l'honneur de Jean Céard
}

\author{
Michele Mastroianni
}

\section{NOTIZIA}

Esculape et Dionysos. Mélanges en l'honneur de Jean Céard, études réunies et éditées par Jean DUPÈBE, Franco GIACONE, Emmanuel NAYA et Anne-Pascale POUEY-Mounou, Genève, Droz, 2008 («Travaux d'Humanisme et Renaissance», n. CDXXXIX) pp. XXXVIII+1176.

1 Render conto in modo esauriente, in una scheda bibliografica, dei settandadue contributi di questi Mélanges è impossibile, tanto più che si tratta di interventi originali e di alto valore scientifico. Si può comunque notare come, a differenza di molte imprese del genere, interessanti ma spesso per la loro stessa natura disorganiche, venga offerto agli studiosi un corpus di articoli costruito in una prospettiva unitaria - quella delle indagini di area cinquecentesca - che è d'altra parte la prospettiva di ricerca dell'insigne seiziémiste qui onorato. Per convincersene è sufficiente dare una scorsa ai titoli dei lavori riuniti: Bénédicte Boudou, Henri Estienne et le stoïcisme dans l'“Apologie pour Hérodote" (pp. 3-14); Marianne cLosson, Une forme inattendue de l'«expérience» au XVI et XVII siècles: la possession démoniaque (pp. 15-26); Marie-Luce DEMONET, Les philosophes obscurs: traits et ombres scotistes à l'époque de Rabelais (pp. 27-47); Francis GOYET, La première phrase des "Essais" (pp. 49-58); Bertrand LEVERGEOIS, Le théâtre d'ombres de Giordano Bruno (pp. 59-66); Jean-François MAILLARD, Réflexions sur une légende: Pléthon, Cosme de Médicis et l'hermétisme ficinien (pp. 67-85); Jean-Claude MARGOLIN, Histoire, nature, prodiges et religion chez Athanase Kircher (pp. 87-109); Emmanuel NAYA, Francisco Sánchez: le médecin et le scepticisme expérimental (pp. 111-129); Nuccio ORDINE, Gli inganni dell'ignoranza: il "Candelaio" tra realtà e apparenza (pp. 131-153); Gilbert SCHRENCK, Pierre de l'Estoile et Montaigne (pp. 155-163); Paola ZAMBELLI, Quelques livres à l'Index chers à Giordano Bruno, ses ceuvres secrètes de magie et un ouvrage qui n'existe pas (pp. 165-174); Stephen BAMFORTH, Médecine et philosophie dans l'œuvre de Nicolas Abraham de La Framboisière (pp. 
177-202); Monica BARSI, Le traitement des sources dans la "Cronique de Pierre Belon du Mans, medecin" (pp. 203-215); Hervé BAUDRY, Pudeur et thérapeutique aux XVI et XVII siècles (pp. 217-230); Donald BEECHER, Sex changes in the Renaissance: a brief medico-literary enquiry (pp. 231-241); Evelyne BERRIOT-SALVADORE, Les œuvres françaises d'André Dulaurens (pp. 243-254); Michel BIDEAUX, La lune, entre thésaurus poétique et encyclopédie pratique (pp. 255-267); Ann BLAIR, Corrections manuscrites et listes d'errata' à la Renaissance (pp. 269-286); Marie-Elisabeth BOUTROUE, De l'index au lexique: recherches sur quelques manuscrits de la Bibl. Vaticane (pp. 287-312); Claude-Françoise BRUNON, La Cigogne, l'Hippopotame et la Huppe: variations hiéroglyphiques (pp. 313-325); Hélène CAZES, Le 'Cogito' de l'anatomiste: "La Dissection des parties du corps humain" par Charles Estienne (pp. 327-341); Marie-Madeleine FRAGONARD, Imaginaire $d u$ monde souterraine et sciences expérimentées (pp. 343-353); Marie-Christine GOMEZ-GÉRAUD, Le voyageur, le médecin et la momie (pp. 355-366); Fernand HALLYN, Paul de Middelbourg, astrologue et astronome (pp. 367-374); Michel JOURDE, Autopsie et réalités sonores au XVI siècle (pp. 375-391); Eva KUSHNER, Tyard et la vérité scientifique (pp. 393-404); John O'BRIEN, Entre théologie et science: la «Chronologia» de Joannes Temporarius (pp. 405-415); Achille OLIVIERI, Gerolamo Cardano: il pensiero medico e il pensiero riformato (pp. 417-425); Isabelle PANTIN, Entre invisible et mystère: les traités de la peste à la Renaissance, discours d'une maladie secrète (pp. 427-442); Laurent PINON, La "Naturalis historia" d'Adam Lonitzer, une œuvre mineure? (pp. 443-457); Jean VIGNES, En attendant Du Pinet: Pierre de Changy et son "Sommaire des Singularitez de Pline" (pp. 459-470; Denis BJAÏ, La disposition du poème épique: le cas Ronsard (pp. 473-483); Sylviane BOKDAM, Un songe d'éclipse dans "L'Encyclie des Secrets de l'Eternité" (pp. 485-502); Keith CAMERON, Regard sur Desportes (pp. 503-510); Richard COOPER, Dolet et Marot jugés par deux poètes contemporains: Jean Binet et Gabriele Simeoni (pp. 511-527); André GENDRE, De Jean Tagaut et de quelques mètres lyriques (pp. 529-542); Rosanna GORRIS CAMOS, "La stella delle maraviglie": un poète et une étoile, la supernova de 1572 (pp. 543-565); MarieDominique LEGRAND, La poétique du "Printemps" d'Agrippa d'Aubigné: aux frontières de l'imaginaire et de l'histoire naturelle (pp. 569-581); Robert MELANÇON, L'Amour ennemi: notes sur l'histoire de la poésie française à la fin du XVI siècle (pp. 583-594); Jan MIERNOWSKI, La science comme objet esthétique dans la poésie française de la Renaissance (pp. 595-605); JeanCharles MONFERRAN, "Faire mes Chaos, et puis mes Mondes": la cosmogonie poétique des premières pages de l'“Art poétique" de J. Peletier du Mans (pp. 607-621); Malcolm QUAINTON, Un texte mineur de Jean-Antoine de Baif? Du nouveau sur «L'Eunuque» (pp. 623-640); Yvette QUENOT, Pape et évêques dans les "Théorèmes" de La Ceppède (pp. 641-653); Jacques BERCHTOLD, Le gosier béant dans le "Gargantua": traitement comique d'une angoisse de dévoration (pp. 657-672); Tom CONLEY, Rébus de Rabelais (pp. 673-682); Franco GIACONE, Deux suppliques inédites de François Rabelais au pape Paul III (pp. 683-705); Isabelle HERSANT, L'imaginaire du vin chez Rabelais. Etude du prologue du "Tiers Livre" (pp. 707-716); Ullrich LANGER, L'opposition et la privation dans "Gargantua" (pp. 717-726); Frank LESTRINGANT, Rabelais, Polydore Vergile et «la fascination des commencements» (pp. 727-740); François BERRIOT, Notes sur le manuscrit d'“Oraisons et meditations" de Marie de Bonnet dame de Sérignan (vers 1601) (pp. 743-751); Nicole CAZAURAN, Abraham et le Diable dans la 'tragédie' de Théodore de Bèze (pp. 753-764); Bruna ConConI, 1526 - La Bible à Lyon: notes sur Jean de Vauzelles traducteur de l'“Histoire évangélique" d'Ammonius (pp. 765-785); Richard CRESCENZO, Numa et la nymphe Egérie: l'approche démonologique de la religion romaine chez Blaise de Vigenère (pp. 787-799); Jean DUPÈBE, Une figure de la Contre-Réforme:l'humaniste 
Giovanni Ferrero (pp. 801-831); Danièle DUPORT, Jardiniers protestants en France au XVI ${ }^{e}$ siècle (pp. 833-846); Max ENGAMMARE, La Bible de Jérôme Bolsec (pp. 847-864); Monique GOSSELINNOAT, Bernanos et Luther (pp. 865-881); Amy C. GRAVES, Dragonnades textuelles: la reprise de la "Lettre à Portus" de Pierre Charpentier dans les "Entretiens touchant l'entreprise du prince d'Orange" de dom Denis de Sainte-Marthe (pp. 883-905); Nadine KUPERTY-TSUR, Des rabbins et des femmes: enjeux culturels d'une argumentation paritaire et bouffonne dans une lettre familière d'Etienne Pasquier (pp. 907-919); Daniel MÉNAGER, Le "Traitté de la vocation" de Pierre de La Place (pp. 921-931); François ROUDAUT, Pierre Nevelet (1554ca.-1610) (pp. 933-963); Cesare VASOLI, Lo scisma e l'eresia nel «De Cardinalatu» di Paolo Cortesi (pp. 965-980); Yvonne BELLENGER, L“'Amas d'Epithetes” de Du Bartas (pp. 983-1000); Marie Madeleine FONTAINE, Barthélemy Aneau et la "Jurisprudentia" (pp. 1001-1012); Mireille HUCHON, Pour une histoire du genre de la nouvelle: "La Vie et actes triumphans de Catherine des bas souhaiz" et "La nouvelle d'un reverend pere en Dieu" de Jean de Luxembourg (1546) (pp. 1013-1028); Catherine MAGNIEN-SIMONIN, Une autobiographie originale de 1590: les "Annales nostrorum laborum" de Guy Coquille (pp. 1029-1044); Michel MAGNIEN, Robert Constantin (ca. 1530-1605), éditeur de Jules-César Scaliger (pp. 1045-1063); Anne-Pascale POUEY-MOUNOU, Grandeur et décadence d'un tout petit genre: les épithétaires de la Renaissance (pp. 1065-1079); Louis-Georges TIN, Un siècle de 'minores'? (pp. 1081-1088); Toshinori UETANI, Jean Martin, traducteur du "Roland Furieux"? (pp. 1089-1109); Véronique ZAERCHER-KECK, Traduire pour éclaircir: Marot, Aneau et quelques colloques d'Erasme (pp. 1111-1123); Oumelbanine ZHIRI, "Sauvages et mahométans" (pp. 1125-1139).

2 I contributi sono stati organizzati in sei sezioni. Nella prima («Philosophie»), l'attenzione è rivolta al configurarsi di alcune linee del pensiero rinascimentale: così, particolare attenzione viene consacrata allo studio dello stoicismo cinquecentesco o alle persistenze della filosofia scolastica medievale, perlomeno a livello lessicale, ai contatti con il pensiero dell'Umanesimo italiano da Ficino a Bruno, al rapporto tra filosofia e scienza. Nella seconda («Sciences»), i saggi concernono soprattutto lo studio della medicina rinascimentale, con un'attenzione particolare per i problemi posti dallo sviluppo, come disciplina, dell'anatomia; ma anche ma anche per l'astronomia e l'astrologia, la zoologia e la storia naturale in genere. Nella terza («Poésie»), il ventaglio degli interventi copre l'arco del Cinquecento, da Marot a La Ceppède, passando per i grandi autori, Ronsard, Baif, Desportes, D’Aubigné, ma anche per i 'minori', con attenzione a problemi di intertestualità e di genere. Nella quarta («Rabelais»), sei contributi fanno il punto delle attuali ricerche rabelaisiane, apportando anche documentazione inedita. Nella quinta («Théologie»), i contributi si occupano dell'ambiente cattolico, con alcune ricerche che recuperano essenzialmente documenti d'archivio, come nel caso della ricostruzione della presenza in Francia dell'umanista piemontese Giovanni Ferrero o nell'illustrazione di un manoscritto inedito (una raccolta di preghiere di Marie de Sérignan) che ci informa della vita quotidiana spirituale e dell'immaginario religioso femminile all'inizio del Seicento; ma soprattutto forniscono documentazione di vario genere sulla cultura, spiritualità, storia delle mentalità protestanti (immaginario tragico, nicodemismo, atteggiamento nei confronti della natura), con attenzione ai rapporti fra Umanesimo e Riforma, in particolare in una prospettiva filologica ed antiquaria negli studi biblici, sempre dimostrando interesse a personaggi minori e poco conosciuti, di cui si traccia un profilo, come nel caso di Pierre Nevelet. Nella sesta («Traduction et histoire littéraire»), si mette in evidenza l'importanza dei cosiddetti minori in un secolo che viene provocatoriamente 
definito siècle de 'minores' (L.-G. Tin), con una perlustrazione fra autori che appartengono al mondo dei traduttori e degli eruditi, o fra opere di autori maggiori che si occupano di traduzione o erudizione, o ancora con un percorso fra generi anch'essi cosiddetti minori. Cosa che non sempre accade in queste imprese celebrative, i curatori hanno apprestato accurati e utili indici dei nomi. 